\title{
Gray and white matter morphology in substance use disorders: a neuroimaging systematic review and meta-analysis
}

Victor Pando-Naude ${ }^{1}$, Sebastian Toxto $\mathbb{B}^{2,3,4}$, Sofia Fernandez-Lozano $\mathbb{1}^{2,5}$, Christine E. Parsons ${ }^{6}$, Sarael Alcauter ${ }^{2}$ and Eduardo A. Garza-Villarreal (10),

\begin{abstract}
Substance use disorders (SUDs) are characterized by a compulsion to seek and consume one or more substances of abuse, with a perceived loss of control and a negative emotional state. Prolonged substance use seems to be associated with morphological changes of multiple neural circuits, in particular the frontal-striatal and limbic pathways. Such neuroadaptations are evident across several substance disorders, but may vary depending on the type of substance, consumption severity and/or other unknown factors. We therefore identified studies investigating the effects of SUDs using volumetric whole-brain voxel-based morphometry (VBM) in gray (GM) and white matter (WM). We performed a systematic review and meta-analysis of VBM studies using the anatomic likelihood estimation (ALE) method implemented in GingerALE (PROSPERO pre-registration CRD42017071222). Sixty studies met inclusion criteria and were included in the final quantitative meta-analysis, with a total of 614 foci, 94 experiments and 4938 participants. We found convergence and divergence in brain regions and volume effects (higher vs. lower volume) in GM and WM depending on the severity of the consumption pattern and type of substance used. Convergent pathology was evident across substances in GM of the insula, anterior cingulate cortex, putamen, and thalamus, and in WM of the thalamic radiation and internal capsule bundle. Divergent pathology between occasional use (cortical pathology) and addiction (cortical-subcortical pathology) provides evidence of a possible top-down neuroadaptation. Our findings indicate particular brain morphometry alterations in SUDs, which may inform our understanding of disease progression and ultimately therapeutic approaches.
\end{abstract}

\section{Introduction}

Substance use disorders (SUDs) refer to a wide range of alterations produced by the consumption of abuse substances or drugs. According to the Diagnostic and Statistical Manual of Mental Disorders (DSM-V) ${ }^{1}$, these substances include: alcohol, caffeine, cannabis, hallucinogens, inhalants, opioids, sedatives, hypnotics and anxiolytics, stimulants, tobacco, and other. About 275

\footnotetext{
Correspondence: Eduardo A. Garza-Villarreal (egarza@comunidad.unam.mx) ${ }^{1}$ Department of Clinical Medicine, Center for Music in the Brain, University of Aarhus, Aarhus, Denmark

${ }^{2}$ Instituto de Neurobiología, Universidad Nacional Autónoma de México (UNAM) campus Juriquilla, Queretaro, Mexico

Full list of author information is available at the end of the article
}

million people worldwide (5.6\% of the global population aged 15-64 years) used substances at least once during $2016^{2}$ and SUDs are recognized as a major public health issue. SUDs affect the reward system, involved in the reinforcement of behaviors and memory, and can lead to chronic use and dependency ${ }^{3}$. Initial substance reward is triggered by dopamine neurons in the ventral tegmental area (VTA), which project to the prefrontal cortex, amygdala and nucleus accumbens (NAc) ${ }^{4,5}$, as well as other ascending monoamine fibers such as norepinephrine and other non-dopaminergic systems within frontal regions ${ }^{6}$.

Additionally, dopaminergic neurons in substantia nigra pars compacta $(\mathrm{SNc})$ project to the dorsal striatum

\section{(c) The Author(s) 2021}

\footnotetext{
(c) (i) Open Access This article is licensed under a Creative Commons Attribution 4.0 International License, which permits use, sharing, adaptation, distribution and reproduction c. in any medium or format, as long as you give appropriate credit to the original author(s) and the source, provide a link to the Creative Commons license, and indicate if changes were made. The images or other third party material in this article are included in the article's Creative Commons license, unless indicated otherwise in a credit line to the material. If material is not included in the article's Creative Commons license and your intended use is not permitted by statutory regulation or exceeds the permitted use, you will need to obtain permission directly from the copyright holder. To view a copy of this license, visit http://creativecommons.org/licenses/by/4.0/.
} 
(nigrostriatal pathway), a pathway implicated in the emergence of habits ${ }^{7}$. A reinforcement effect seems to depend on dopaminergic signaling in the NAc, and chronic use has been associated to neuroadaptations of the striato-thalamo-cortical (prefrontal cortex, orbitofrontal cortex and the anterior cingulate cortex) and limbic pathways (amygdala and hippocampus) ${ }^{4,5}$, especially in individuals who may be vulnerable due to genetic and/or environmental factors ${ }^{8}$. Other endogenous systems, such as the opioid and cannabinoid systems, may contribute to the reinforcement effect by modulating hedonic responses or inhibiting negative affective states ${ }^{9}$.

Substance-induced neuroadaptations are similar to synaptic changes associated with learning, including changes in dendritic morphology and ionotropic glutamate receptors (e.g., AMPA/NMDA), which result in long-term potentiation (LTP) and long-term depression $(\mathrm{LTD})^{10,11}$. Notably, the link between repeated dopaminergic signaling and neuroadaptations is yet unclear, and causality should be interpreted with caution. These neuroadaptations result in pathological changes in brain morphology, that seem to be salient enough to be observed macroscopically with magnetic resonance imaging (MRI), as shown by neuroimaging studies in humans and animal models ${ }^{12,13}$.

Neuroimaging studies using MRI have shown alterations in gray and white matter in SUDs ${ }^{14,15}$. However, the involved regions vary widely and seem to depend on the type of substance, the consumption severity, the age of first use, the total time of usage, and other associated comorbidities. Morphometric studies investigating the effects of SUDs using volumetric measures such as voxelbased morphometry (VBM), have reported both lower and higher volume in cortical and subcortical gray matter $(\mathrm{GM})^{16,17}$ and white matter $(\mathrm{WM})^{18,19}$. For example, alcohol use disorder (AUD) studies have shown lower GM volume of the amygdala, insula, cingulate gyrus, orbitofrontal gyrus and thalamus ${ }^{14}$, while tobacco use disorder (TUD) studies have shown lower GM volume in thalamus, cingulate gyrus, prefrontal cortex, and cerebellum ${ }^{20}$. Cocaine use disorder (CUD) studies have shown lower GM volume in thalamus, insula, orbitofrontal cortex, anterior cingulate cortex, superior temporal cortex, and cerebellum $^{21}$. Conversely, other studies of these same substances have shown higher GM volume in putamen and other nuclei of the basal ganglia ${ }^{22,23}$. Similarly, WM studies have shown different substances affecting different areas in distinct manners. For example, studies of AUD, TUD, and CUD have shown lower volume of WM in the corticospinal tract, thalamic radiations, and the corpus callosum $^{20,24-26}$. Overall, the structural pathology seems to be both convergent and divergent in terms of localization between studies.
Given these findings, it is unclear how SUDs affect brain morphology and how to differentiate between distinct changes caused by substance toxicity and substance dependency ${ }^{27}$. Potential reasons for the variability in findings may include: (1) study definitions (substance use disorder vs addiction vs dependency), (2) polysubstance use, (3) the substance user characteristics, such as age or time of substance use, and (4) methodological differences between morphometric studies (i.e., software used). Thus, a meta-analysis of brain imaging studies provides an opportunity to better understand the mechanisms by which SUDs affect brain morphology, of great interest for treatment follow-up as well as potential marker of therapy success. In this systematic review and meta-analysis of VBM studies, we aimed at finding the overall effect of SUDs in GM and WM volume, and to differentiate the possible mechanisms behind such effects by means of subgroup analyses of the type of substance, consumption severity, age and associated comorbidities.

\section{Materials and methods \\ Literature search, screening, and extraction}

This systematic review and meta-analysis followed procedures from the Cochrane Handbook for Systematic Reviews $^{28}$, and from the Center for Reviews and Dissemination (https://www.york.ac.uk/crd/). The review protocol was pre-registered in PROSPERO (CRD42017071222). This review was carried in accordance with the PRISMA ${ }^{29}$. We conducted a systematic literature search in PubMed, Scopus and PsycInfo, using both keywords and MeSH terms for articles published up to August 10th, 2020. No restrictions were placed on study design, but in order to be eligible for inclusion, the studies must have reported whole-brain VBM analyses. Screening and data extraction were performed using the Covidence tool ${ }^{30}$. The main outcome to extract was any change in gray and/or white matter analyzed using VBM, in stereotactic coordinates, comparing a substance user group and a healthy control group (details in Supplementary information).

\section{Quality assessment of MRI studies}

Criteria for MRI quality reporting was selected from a set of guidelines for the standardized reporting of MRI studies $^{31-33}$. Such guidelines dictate a more consistent and coherent policy for the reporting of MRI methods to ensure that methods can be understood and replicated.

\section{Analysis and meta-analytic technique}

Statistically significant foci from between-group contrasts were extracted and recorded for each study. Where necessary, coordinates were converted from Talairach coordinates to MNI space using the Lancaster transform (icbm2tal) incorporated in GingerALE ${ }^{34,35}$. All meta- 
analyses were performed using anatomic likelihood estimation (ALE), implemented in GingerALE, in Brain$\mathrm{Map}^{36}$. This method extracts the coordinates from the included studies and tests for anatomical consistency and concordance between the studies. The coordinates are weighted according to the size of the sample (number of participants), and these weightings contribute to form estimates of anatomic likelihood estimation for each intracerebral voxel on a standardized map. This approach treats anatomic foci (input) not as single points, but as spatial probability distributions centered at the given coordinates. Therefore, the algorithm tests to what extent the spatial locations of the foci correlate across independently conducted MRI studies investigating the same construct, and assesses them against a null-distribution of random spatial association between experiments ${ }^{37}$. Statistical significance of the ALE scores was determined by a permutation test using cluster-level inference at $p<$ 0.05 (FWE). As we did not impose any minimum cluster size of supra-threshold voxels, small volume clusters should be interpreted with caution.

The primary outcome was morphological brain differences measured by VBM between substance users (SU) and healthy controls $(\mathrm{HC})$, pooling all substances together, to examine comprehensively the structural changes associated with SUD. To test the directionality of the primary outcome, we pooled coordinates reporting higher volume with substance use $(\mathrm{HC}<\mathrm{SU})$ and lower volume with substance use $(\mathrm{SU}<\mathrm{HC})$. Pre-registered subgroup analyses included age of substance users (adolescents vs. adults), consumption severity (addiction vs. long-term use vs. occasional use), type of substance (alcohol vs. tobacco vs. cannabis vs. cocaine vs. stimulants vs. opioids vs. ketamine vs. polysubstance; the latter refers to studies that combined substances into one main effect from the contrast SU vs. HC) and associated comorbidities (pure vs. dual). Finally, subgroups were tested for similarity (conjunction) and difference (subtraction) in a contrast analysis. All meta-analyses were conducted separately for GM and WM. We use "addiction" as a synonym for SUD that includes dependency, as the latter definition is fairly recent ${ }^{1}$. Additionally, addiction, long-term use and occasional use could also be regarded as severe-SUD, moderate-SUD, and mild-SUD, respectively.

We conducted meta-analytic connectivity modeling $(\mathrm{MACM})^{38}$ to analyse co-activation patterns of regionsof-interest (ROI) resulting from the primary outcomes, aiming to functionally segregate each region's putative contribution to behavioral domains ${ }^{39}$. Co-activation analyses were performed using Sleuth ${ }^{40}$ and GingerALE from the BrainMap database.

The meta-analytic results (ALE maps) were visualized using Mango on the MNI152 $1 \mathrm{~mm}$ standard brain, and resulting coordinates were cross-referenced to the
Harvard-Oxford Cortical and Subcortical Atlas and the Juelich Histological Atlas via NeuroVault ${ }^{41}$ and FSLeyes ${ }^{42}$, respectively.

Finally, we performed the Fail-Safe $\mathrm{N}$ analysis (FSN) ${ }^{43}$ as a measure of robustness against potential publication bias. It refers to the amount of contra-evidence that can be added to a meta-analysis before the results change, and can be obtained for each cluster that survives thresholding in an ALE meta-analysis. A higher FSN indicates more stable results and hence a higher robustness.

\section{Results}

A total of 1095 records were identified through database searching, and after removing duplicates, 584 records were initially screened by title and abstract. A total of 584 articles were assessed for eligibility in the full-text screening stage. From these, 60 studies fulfilled criteria for eligibility and were included in both the qualitative and quantitative analyses (Supplementary Fig. 1).

\section{Characteristics of studies}

The characteristics of studies included in the metaanalysis are shown in Table 1. Sixty studies met inclusion criteria and were included in the final quantitative metaanalysis, with a total of 614 foci and 94 experiments. The total number of participants was 4938 , with $49.2 \%$ substance users (SU) and 50.8\% heathy controls (HC). For the SU subsample, $64 \%$ in the addiction group (A), $7 \%$ on the long-term use group (LT), and $29 \%$ on the occasional use group $(\mathrm{O})$. Alcohol was the main substance of interest in $20 \%$ of studies, tobacco $22 \%$, cocaine $12 \%$, cannabis $12 \%$, opioids $12 \%$, stimulants $6 \%$, ketamine $2 \%$, and polysubstance use $14 \%$. SUD was evaluated by a psychiatrist in $27 \%$ of studies, psychologist $7 \%$, clinician $2 \%$, while $64 \%$ failed to report the evaluator. The DSM-IV was used in $70 \%$ of studies, DSM-V $7 \%$, while $23 \%$ failed to report the tool used to diagnose substance use disorder. All of the studies reported change in GM volume (100\%), while 15 studies $(25 \%)$ reported change in WM volume.

Neuroimaging data was acquired in either $1.5 \mathrm{~T}(43 \%)$, or $3 \mathrm{~T}(57 \%)$ MRI scanners. Half of the studies were conducted in a Siemens MRI system, others were general electric (30\%), Phillips (18\%), and Bruker (2\%). Most of the T1w-structural images were acquired using magnetization-prepared rapid acquisition with gradient echo sequence (MPRAGE), in 25 studies (42\%), and $1 \mathrm{~mm}^{3}$-voxel size in 36 studies (60\%). VBM analyses were conducted in either $\mathrm{SPM}^{44}(82 \%), \mathrm{FSL}^{42}(18 \%)$, or $\mathrm{AFNI}^{45}$ (2\%) (Supplementary Table 1).

\section{MRI quality}

MRI quality of the included studies in the meta-analysis was assessed by a set of guidelines for the standardized reporting of MRI studies ${ }^{31-33}$ (Supplementary Table 2). 


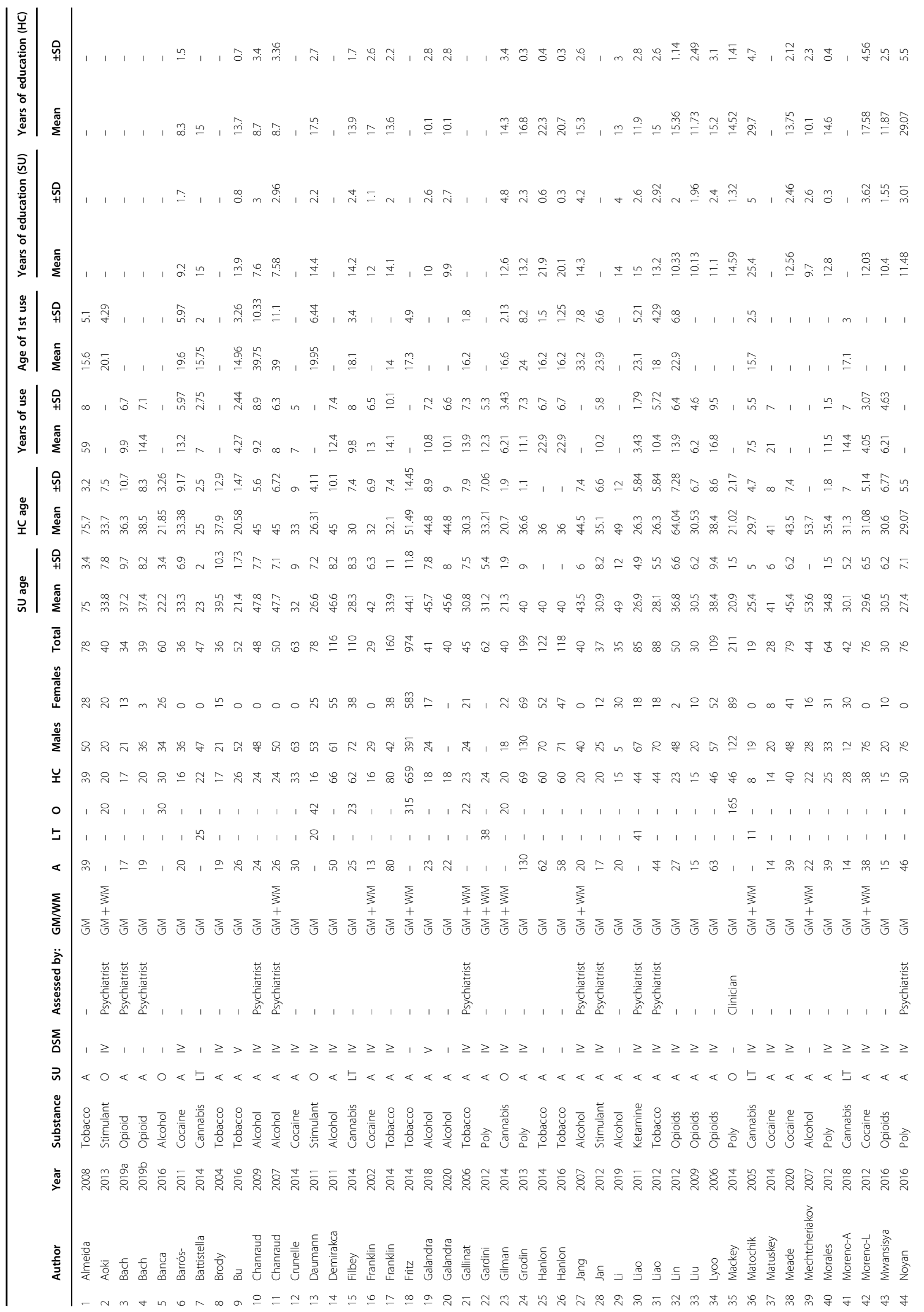




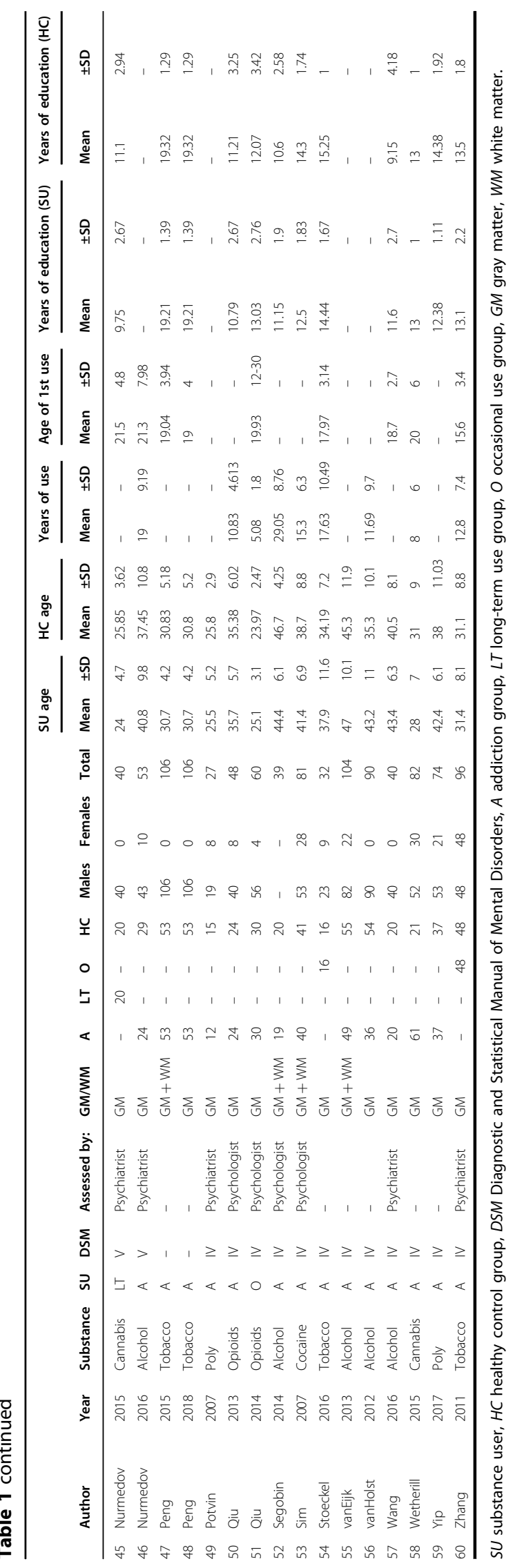

All studies reported their MRI design, software package and image acquisition, processing and analyses. Overall, good MRI practices were performed in the included studies.

\section{Primary outcome}

The primary outcome was brain morphological differences measured by VBM between $\mathrm{SU}$ and $\mathrm{HC}$, pooling all substances together, and defined as higher or lower volume. First, we included all substances and all reported coordinates and found three clusters in GM: right anterior cingulate cortex, left putamen and left thalamus; and one cluster in WM: right anterior thalamic radiation. Second, the comparison $\mathrm{SU}<\mathrm{HC}$ (lower volume with use) resulted in three GM clusters: right anterior cingulate cortex, left thalamus and left insula; and one WM cluster: right anterior thalamic radiation. Finally, the comparison $\mathrm{HC}<$ SU (higher volume with use) resulted in one GM cluster: left putamen; and three WM clusters: right corticospinal tract, left superior longitudinal fasciculus and left optic radiation (Fig. 1 and Table 2).

\section{Subgroup analyses}

Pre-hoc subgroup analyses included (1) age of substance user: adolescents vs. adults; (2) consumption severity: addiction vs. long-term use vs. occasional use; (3) type of substance: alcohol vs. tobacco vs cannabis vs. cocaine vs. stimulants vs. opioids vs. ketamine, and papers that pooled together substances which we termed polysubstance; and (4) associated comorbidities: single vs. multiple. Age and comorbidity subgroups resulted in insufficient experiments (foci) to conduct an ALE analysis $(<15)$. However, we found significant ALE maps in the subgroups consumption severity and type of substance (Fig. 2).

\section{Subgroup analysis by type consumption}

The first subgroup meta-analysis reported ALE maps of substance users (SU) against healthy controls $(\mathrm{HC})$, by type of consumption severity (addiction vs. long-term use vs. occasional use). We found significant ALE maps showing lower GM and WM volumes across all types of consumption. Additionally, higher GM volumes were also shown across all types of consumption, and higher WM only in long-term use (Fig. 2 and Supplementary Table 3).

We conducted contrast analyses between the ALE maps of each subgroup, to determine similarity (conjunction) and/or difference (subtraction) of affected brain regions between the types of consumption (Fig. 3 and Supplementary Table 4). Addiction and long-term use were both associated with lower GM volume of the thalamus but differ in terms of lower GM of red nucleus, substantia nigra, and putamen. These results support the idea that the thalamus is affected across all levels of SUD severity, 


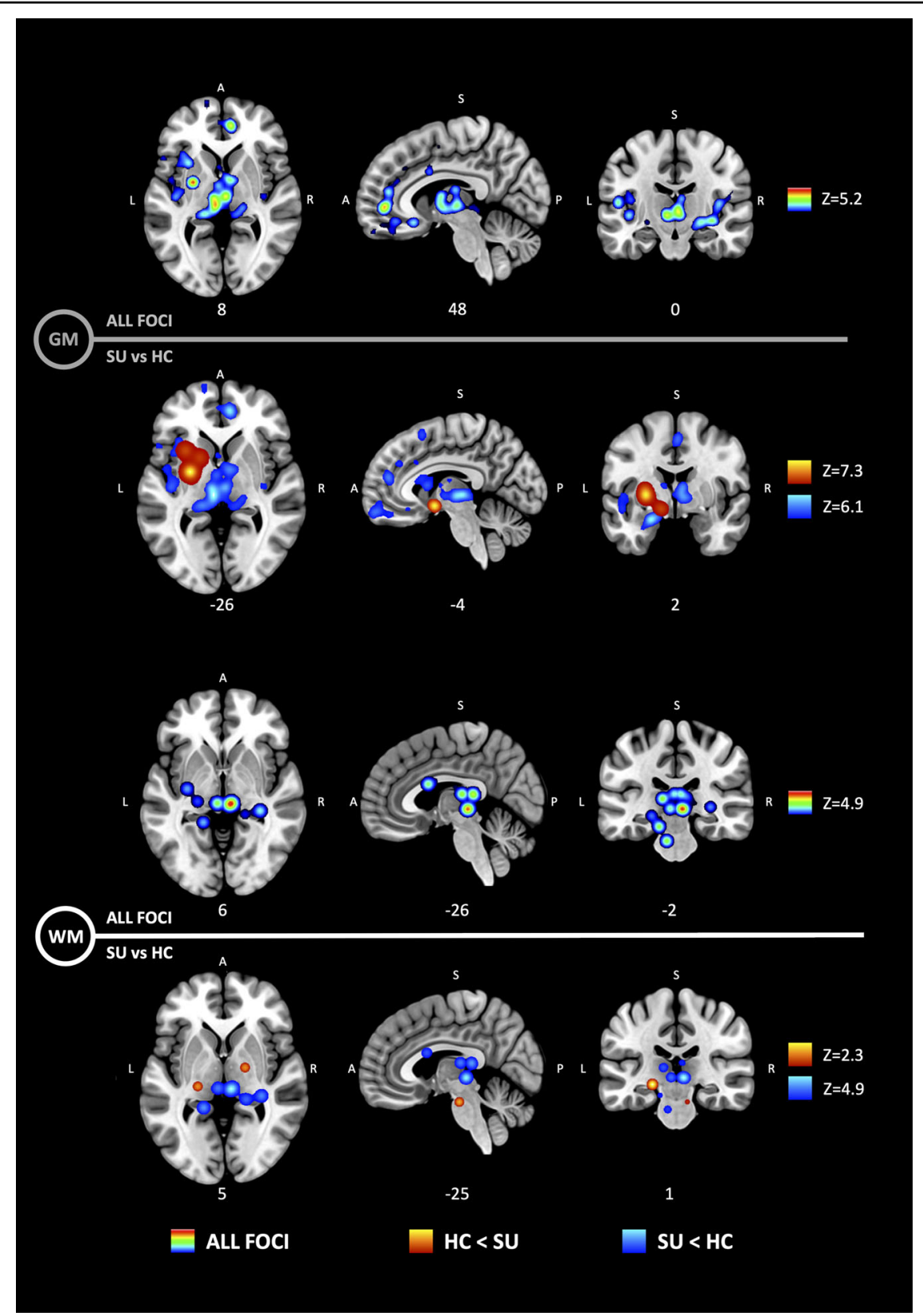

Fig. 1 Anatomic likelihood estimation meta-analytic results for studies comparing brain morphological changes between SU and HC, at cluster level inference $\boldsymbol{p}<\mathbf{0 . 0 5}$ (FWE). The primary outcome included GM (top) and WM (bottom) volumetric alterations in SUDs. HC $<$ SU = higher volume with use; $\mathrm{SU}<\mathrm{HC}=$ lower volume with use. Significant ALE maps show lower volume in thalamus, insula and anterior cingulate cortex in GM, and thalamic radiations in WM; and higher volume in putamen GM, and corticospinal WM tract. Such results support the idea that the entire limbic loop of the basal ganglia shows neuroadaptations produced by SUD. Z, peak Z-value.

and future research should focus on the correlation between SUD progression and the volume/form of the thalamus, as its morphology may predict severity of the disease, and/or monitor the efficacy of treatments and therapies. Addiction and occasional use both show higher volume of the globus pallidus, while differ in lower volume of fronto-temporal areas including the medial frontal gyrus, anterior cingulate cortex and superior temporal gyrus, supporting cortical alterations in occasional use. Finally, long-term use and occasional use share higher volume of somatomotor cortices, due to possible drug intoxication. In terms of WM, addiction and longterm use share lower volume of the anterior thalamic radiations and the corpus callosum, suggesting also a probable correlation between the progression of SUD and the severity in WM structural alteration. 
Table 2 Anatomic likelihood estimation meta-analytic results for studies comparing brain morphological changes between SU and HC at cluster level inference $p<0.05$ (FWE).

\begin{tabular}{|c|c|c|c|c|c|c|c|c|}
\hline \multirow[t]{2}{*}{ Cluster number } & \multirow[t]{2}{*}{ Volume $\left(\mathrm{mm}^{3}\right)$} & \multicolumn{3}{|c|}{ MNI coordinates } & \multirow[t]{2}{*}{ ALE } & \multirow[t]{2}{*}{$P$} & \multirow[t]{2}{*}{$z$} & \multirow[t]{2}{*}{ Label (Side region BA) } \\
\hline & & $x$ & $y$ & $z$ & & & & \\
\hline \multicolumn{9}{|l|}{ a. GM: All foci } \\
\hline \multirow[t]{24}{*}{1} & 23,768 & 8 & 48 & 0 & $3 E-02$ & $1 \mathrm{E}-07$ & 5.2 & R Anterior cingulate cortex BA32 \\
\hline & & 0 & 48 & -16 & $3 E-02$ & $8 \mathrm{E}-07$ & 4.8 & L Anterior cingulate cortex BA32 \\
\hline & & -10 & 58 & -16 & $3 \mathrm{E}-02$ & $8 \mathrm{E}-06$ & 4.3 & L Superior frontal gyrus BA10 \\
\hline & & -2 & 14 & 58 & $3 E-02$ & $8 \mathrm{E}-06$ & 4.3 & L Superior frontal gyrus BA6 \\
\hline & & -2 & 36 & 30 & $2 \mathrm{E}-02$ & $2 \mathrm{E}-05$ & 4.1 & L Medial frontal gyrus BA6 \\
\hline & & 0 & 24 & 46 & $2 \mathrm{E}-02$ & $3 \mathrm{E}-05$ & 4.1 & L Medial frontal gyrus BA6 \\
\hline & & -4 & 46 & 16 & $2 \mathrm{E}-02$ & $3 \mathrm{E}-05$ & 4.0 & L Medial frontal gyrus BA9 \\
\hline & & -2 & 20 & 32 & $2 \mathrm{E}-02$ & $8 \mathrm{E}-05$ & 3.8 & L Anterior cingulate cortex BA32 \\
\hline & & 8 & 22 & -14 & $2 \mathrm{E}-02$ & $1 \mathrm{E}-04$ & 3.7 & R Anterior cingulate cortex BA32 \\
\hline & & 4 & 44 & 14 & $2 \mathrm{E}-02$ & $2 \mathrm{E}-04$ & 3.6 & R Anterior cingulate cortex BA32 \\
\hline & & 16 & 20 & -16 & $2 E-02$ & $3 E-04$ & 3.4 & R Orbitofrontal cortex BA47 \\
\hline & & 2 & 0 & 52 & $2 \mathrm{E}-02$ & $5 E-04$ & 3.3 & L Medial frontal gyrus BA6 \\
\hline & & 6 & 38 & -14 & $2 \mathrm{E}-02$ & $2 \mathrm{E}-03$ & 3.0 & $\mathrm{R}$ Anterior cingulate cortex BA32 \\
\hline & & 6 & 8 & 30 & $1 \mathrm{E}-02$ & $2 \mathrm{E}-03$ & 2.8 & R Anterior cingulate cortex BA24 \\
\hline & & -14 & 64 & -2 & $1 \mathrm{E}-02$ & $7 \mathrm{E}-03$ & 2.4 & L Medial frontal gyrus BA10 \\
\hline & & -4 & 56 & -6 & $1 \mathrm{E}-02$ & $8 \mathrm{E}-03$ & 2.4 & L Medial frontal gyrus BA10 \\
\hline & & -14 & 68 & 0 & $1 \mathrm{E}-02$ & $1 \mathrm{E}-02$ & 2.3 & L Superior frontal gyrus BA10 \\
\hline & & -2 & 50 & 28 & $1 E-02$ & $1 E-02$ & 2.2 & L Medial frontal gyrus BA9 \\
\hline & & -2 & 62 & -6 & $1 E-02$ & $2 \mathrm{E}-02$ & 2.1 & L Medial frontal gyrus BA10 \\
\hline & & 6 & 58 & -22 & $1 \mathrm{E}-02$ & $2 \mathrm{E}-02$ & 2.1 & R Medial frontal gyrus BA11 \\
\hline & & 14 & 38 & -26 & $1 \mathrm{E}-02$ & $2 \mathrm{E}-02$ & 2.1 & R Inferior frontal gyrus BA11 \\
\hline & & 28 & 20 & -16 & $1 E-02$ & $2 E-02$ & 2.1 & R Claustrum \\
\hline & & -12 & 14 & 66 & $1 \mathrm{E}-02$ & $2 \mathrm{E}-02$ & 2.1 & L Superior frontal gyrus BA6 \\
\hline & & 8 & 8 & 38 & $1 \mathrm{E}-02$ & $2 \mathrm{E}-02$ & 2.0 & R Cingulate gyrus BA24 \\
\hline \multirow[t]{12}{*}{2} & 23,256 & -6 & -24 & 0 & $4 \mathrm{E}-02$ & $4 \mathrm{E}-08$ & 5.4 & L Thalamus \\
\hline & & 2 & -16 & 4 & $3 \mathrm{E}-02$ & $6 \mathrm{E}-07$ & 4.8 & L Thalamus MDN \\
\hline & & 34 & -14 & -6 & $2 \mathrm{E}-02$ & $1 \mathrm{E}-05$ & 4.2 & R Putamen \\
\hline & & -16 & -32 & 0 & $2 \mathrm{E}-02$ & $4 \mathrm{E}-05$ & 3.9 & L Thalamus \\
\hline & & 8 & -2 & 0 & $2 \mathrm{E}-02$ & $5 E-05$ & 3.9 & R Thalamus \\
\hline & & 6 & -2 & 8 & $2 \mathrm{E}-02$ & $7 \mathrm{E}-05$ & 3.8 & R Thalamus \\
\hline & & 40 & -18 & 10 & $2 \mathrm{E}-02$ & $1 \mathrm{E}-04$ & 3.7 & R Insula BA13 \\
\hline & & 24 & -16 & -6 & $2 \mathrm{E}-02$ & $1 \mathrm{E}-04$ & 3.7 & R Lateral globus pallidus \\
\hline & & -4 & -8 & 0 & $2 \mathrm{E}-02$ & $2 E-04$ & 3.5 & L Thalamus \\
\hline & & 18 & -26 & 4 & $2 \mathrm{E}-02$ & $3 \mathrm{E}-04$ & 3.5 & R Thalamus \\
\hline & & -6 & 10 & 10 & $2 \mathrm{E}-02$ & $4 \mathrm{E}-04$ & 3.3 & L Caudate body \\
\hline & & 6 & -14 & 14 & $2 E-02$ & $5 E-04$ & 3.3 & R Thalamus MDN \\
\hline
\end{tabular}


Table 2 continued

\begin{tabular}{|c|c|c|c|c|c|c|c|c|}
\hline \multirow[t]{2}{*}{ Cluster number } & \multirow[t]{2}{*}{ Volume $\left(\mathrm{mm}^{3}\right)$} & \multicolumn{3}{|c|}{ MNI coordinates } & \multirow[t]{2}{*}{ ALE } & \multirow[t]{2}{*}{$P$} & \multirow[t]{2}{*}{$z$} & \multirow[t]{2}{*}{ Label (Side region BA) } \\
\hline & & $x$ & $y$ & $z$ & & & & \\
\hline & & 48 & -28 & 18 & $2 \mathrm{E}-02$ & $5 E-04$ & 3.3 & R Insula BA13 \\
\hline & & 16 & -42 & -10 & $2 \mathrm{E}-02$ & $2 \mathrm{E}-03$ & 2.9 & R Culmen \\
\hline & & -12 & -4 & 12 & $2 \mathrm{E}-02$ & $2 \mathrm{E}-03$ & 2.9 & L Thalamus VAN \\
\hline & & -6 & 14 & 16 & $1 \mathrm{E}-02$ & $6 \mathrm{E}-03$ & 2.5 & L Caudate body \\
\hline & & -4 & 10 & 2 & $1 E-02$ & $1 \mathrm{E}-02$ & 2.2 & L Caudate body \\
\hline & & -8 & -12 & 14 & $1 E-02$ & $2 \mathrm{E}-02$ & 2.0 & L Thalamus \\
\hline \multirow[t]{25}{*}{3} & 22,432 & -26 & -4 & 4 & $4 \mathrm{E}-02$ & $1 \mathrm{E}-08$ & 5.6 & L Putamen \\
\hline & & -52 & -16 & 12 & $2 \mathrm{E}-02$ & $5 \mathrm{E}-05$ & 3.9 & L Transverse temporal gyrus BA41 \\
\hline & & -44 & -10 & -6 & $2 \mathrm{E}-02$ & $6 \mathrm{E}-05$ & 3.8 & L Insula BA13 \\
\hline & & -30 & 14 & 2 & $2 \mathrm{E}-02$ & $2 \mathrm{E}-04$ & 3.5 & L Claustrum \\
\hline & & -42 & -16 & 0 & $2 \mathrm{E}-02$ & $3 \mathrm{E}-04$ & 3.4 & L Insula BA13 \\
\hline & & -26 & -14 & -6 & $2 \mathrm{E}-02$ & $9 \mathrm{E}-04$ & 3.1 & L Lateral globus pallidus \\
\hline & & -16 & 18 & -14 & $2 \mathrm{E}-02$ & $1 \mathrm{E}-03$ & 3.1 & L Putamen \\
\hline & & -40 & 12 & -2 & $1 E-02$ & $3 \mathrm{E}-03$ & 2.8 & L Insula BA13 \\
\hline & & -10 & 28 & -14 & $1 E-02$ & $3 \mathrm{E}-03$ & 2.7 & L Caudate head \\
\hline & & -40 & -18 & 14 & $1 \mathrm{E}-02$ & $3 \mathrm{E}-03$ & 2.7 & L Insula BA13 \\
\hline & & -48 & 2 & -8 & $1 E-02$ & $5 \mathrm{E}-03$ & 2.6 & L Superior temporal gyrus BA22 \\
\hline & & -24 & 16 & -8 & $1 \mathrm{E}-02$ & $6 \mathrm{E}-03$ & 2.5 & L Putamen \\
\hline & & -36 & 8 & -10 & $1 \mathrm{E}-02$ & $6 \mathrm{E}-03$ & 2.5 & L Claustrum \\
\hline & & -28 & 8 & -8 & $1 E-02$ & $8 \mathrm{E}-03$ & 2.4 & L Putamen \\
\hline & & -34 & -8 & -26 & $1 E-02$ & $8 \mathrm{E}-03$ & 2.4 & L Amygdala \\
\hline & & -46 & -2 & 2 & $1 \mathrm{E}-02$ & $1 \mathrm{E}-02$ & 2.3 & L Insula BA13 \\
\hline & & -42 & 12 & 6 & $1 \mathrm{E}-02$ & $1 \mathrm{E}-02$ & 2.3 & L Insula BA13 \\
\hline & & -54 & 18 & 2 & $1 E-02$ & $1 E-02$ & 2.3 & L Precentral gyrus BA44 \\
\hline & & -48 & 16 & -2 & $1 \mathrm{E}-02$ & $1 \mathrm{E}-02$ & 2.3 & L Insula BA13 \\
\hline & & -64 & -4 & -16 & $1 \mathrm{E}-02$ & $1 \mathrm{E}-02$ & 2.2 & L Middle temporal gyrus BA21 \\
\hline & & -58 & -4 & -16 & $1 E-02$ & $1 E-02$ & 2.2 & L Middle temporal gyrus BA21 \\
\hline & & -30 & 18 & -18 & $1 E-02$ & $1 E-02$ & 2.2 & L Inferior frontal gyrus BA47 \\
\hline & & -38 & -28 & 10 & $1 \mathrm{E}-02$ & $2 \mathrm{E}-02$ & 2.1 & L Transverse temporal gyrus BA41 \\
\hline & & -26 & -10 & -18 & $1 \mathrm{E}-02$ & $2 \mathrm{E}-02$ & 2.1 & L Amygdala \\
\hline & & -32 & 16 & -26 & $1 \mathrm{E}-02$ & $2 \mathrm{E}-02$ & 2.1 & L Inferior frontal gyrus BA47 \\
\hline \multicolumn{9}{|c|}{ b. GM: lower volume with use $(S U<H C)$} \\
\hline \multirow[t]{6}{*}{1} & 25,360 & 8 & 48 & 0 & $3 \mathrm{E}-02$ & $8 \mathrm{E}-08$ & 5.3 & R Anterior cingulate BA32 \\
\hline & & 0 & 48 & -16 & $3 \mathrm{E}-02$ & $6 \mathrm{E}-07$ & 4.9 & R Anterior cingulate BA32 \\
\hline & & -10 & 58 & -16 & $3 E-02$ & $6 \mathrm{E}-06$ & 4.4 & L Superior frontal gyrus BA10 \\
\hline & & -2 & 14 & 58 & $3 \mathrm{E}-02$ & $6 \mathrm{E}-06$ & 4.4 & L Superior frontal gyrus BA6 \\
\hline & & -2 & 36 & 30 & $2 \mathrm{E}-02$ & $1 \mathrm{E}-05$ & 4.2 & L Medial frontal gyrus BA6 \\
\hline & & 0 & 24 & 46 & $2 \mathrm{E}-02$ & $2 \mathrm{E}-05$ & 4.1 & R Medial frontal gyrus BA6 \\
\hline
\end{tabular}


Table 2 continued

\begin{tabular}{|c|c|c|c|c|c|c|c|c|}
\hline \multirow[t]{2}{*}{ Cluster number } & \multirow[t]{2}{*}{ Volume $\left(\mathrm{mm}^{3}\right)$} & \multicolumn{3}{|c|}{ MNI coordinates } & \multirow[t]{2}{*}{ ALE } & \multirow[t]{2}{*}{$P$} & \multirow[t]{2}{*}{$Z$} & \multirow[t]{2}{*}{ Label (Side region BA) } \\
\hline & & $x$ & $y$ & $z$ & & & & \\
\hline & & -4 & 46 & 16 & $2 \mathrm{E}-02$ & $2 \mathrm{E}-05$ & 4.1 & L Medial frontal gyrus BA9 \\
\hline & & -2 & 20 & 32 & $2 \mathrm{E}-02$ & $6 \mathrm{E}-05$ & 3.8 & L Cingulate gyrus BA32 \\
\hline & & 8 & 22 & -14 & $2 \mathrm{E}-02$ & $1 E-04$ & 3.7 & R Anterior cingulate BA32 \\
\hline & & 4 & 44 & 14 & $2 \mathrm{E}-02$ & $1 E-04$ & 3.7 & R Anterior cingulate BA32 \\
\hline & & 2 & 0 & 52 & $2 \mathrm{E}-02$ & $4 \mathrm{E}-04$ & 3.3 & R Medial frontal gyrus BA6 \\
\hline & & 6 & 38 & -14 & $2 \mathrm{E}-02$ & $1 E-03$ & 3.0 & R Anterior cingulate BA32 \\
\hline & & 6 & 8 & 30 & $1 \mathrm{E}-02$ & $2 \mathrm{E}-03$ & 2.9 & R Cingulate gyrus BA24 \\
\hline & & -10 & 30 & -14 & $1 E-02$ & $3 E-03$ & 2.8 & L Anterior cingulate BA24 \\
\hline & & -14 & 64 & -2 & $1 E-02$ & $6 \mathrm{E}-03$ & 2.5 & L Medial frontal gyrus BA10 \\
\hline & & -4 & 56 & -6 & $1 \mathrm{E}-02$ & $7 E-03$ & 2.5 & L medial frontal gyrus BA10 \\
\hline & & -14 & 68 & 0 & $1 \mathrm{E}-02$ & $9 \mathrm{E}-03$ & 2.4 & L Superior frontal gyrus BA10 \\
\hline & & 2 & 36 & -30 & $1 \mathrm{E}-02$ & $1 E-02$ & 2.3 & R Rectal gyrus BA11 \\
\hline & & -2 & 50 & 28 & $1 \mathrm{E}-02$ & $1 E-02$ & 2.3 & L Medial frontal gyrus BA9 \\
\hline & & -2 & 62 & -6 & $1 \mathrm{E}-02$ & $1 E-02$ & 2.2 & L Medial frontal gyrus BA10 \\
\hline & & 6 & 58 & -22 & $1 \mathrm{E}-02$ & $1 \mathrm{E}-02$ & 2.2 & R Medial frontal gyrus BA11 \\
\hline & & 14 & 38 & -26 & $1 \mathrm{E}-02$ & $1 E-02$ & 2.2 & R Inferior frontal gyrus BA11 \\
\hline & & -12 & 14 & 66 & $1 \mathrm{E}-02$ & $2 E-02$ & 2.1 & L Superior Frontal Gyrus BA6 \\
\hline & & 28 & 20 & -14 & $1 \mathrm{E}-02$ & $2 \mathrm{E}-02$ & 2.1 & R Claustrum \\
\hline & & 8 & 8 & 38 & $1 \mathrm{E}-02$ & $2 \mathrm{E}-02$ & 2.1 & R Cingulate gyrus BA24 \\
\hline \multirow[t]{19}{*}{2} & 22,824 & -6 & -24 & 0 & $4 \mathrm{E}-02$ & $3 E-08$ & 5.4 & L Thalamus \\
\hline & & 2 & -16 & 4 & $3 E-02$ & $2 \mathrm{E}-06$ & 4.6 & R Thalamus \\
\hline & & -16 & -32 & 0 & $2 \mathrm{E}-02$ & $3 \mathrm{E}-05$ & 4.0 & L Thalamus \\
\hline & & 8 & -2 & 0 & $2 E-02$ & $4 \mathrm{E}-05$ & 3.9 & R Thalamus \\
\hline & & 6 & -2 & 8 & $2 \mathrm{E}-02$ & $5 E-05$ & 3.9 & R Thalamus \\
\hline & & 32 & -16 & -6 & $2 \mathrm{E}-02$ & $6 \mathrm{E}-05$ & 3.9 & R Lentiform nucleus \\
\hline & & 40 & -18 & 10 & $2 \mathrm{E}-02$ & $8 \mathrm{E}-05$ & 3.8 & R Insula BA13 \\
\hline & & 24 & -16 & -6 & $2 E-02$ & $8 \mathrm{E}-05$ & 3.8 & R Lentiform nucleus \\
\hline & & 18 & -26 & 4 & $2 \mathrm{E}-02$ & $2 E-04$ & 3.5 & R Thalamus \\
\hline & & -4 & -6 & 0 & $2 \mathrm{E}-02$ & $3 \mathrm{E}-04$ & 3.4 & L Thalamus \\
\hline & & -6 & 10 & 10 & $2 \mathrm{E}-02$ & $3 E-04$ & 3.4 & L Caudate \\
\hline & & 6 & -14 & 14 & $2 E-02$ & $4 \mathrm{E}-04$ & 3.4 & R Thalamus \\
\hline & & 48 & -28 & 18 & $2 \mathrm{E}-02$ & $4 \mathrm{E}-04$ & 3.3 & R Insula BA13 \\
\hline & & -12 & -4 & 12 & $2 \mathrm{E}-02$ & $2 E-03$ & 3.0 & L Thalamus \\
\hline & & -6 & 14 & 16 & $1 E-02$ & $5 E-03$ & 2.6 & L Caudate \\
\hline & & 0 & -10 & 24 & $1 \mathrm{E}-02$ & $1 E-02$ & 2.3 & R Cingulate gyrus BA23 \\
\hline & & -4 & 10 & 2 & $1 \mathrm{E}-02$ & $1 \mathrm{E}-02$ & 2.3 & L Caudate \\
\hline & & 20 & -20 & 14 & $1 E-02$ & $1 E-02$ & 2.2 & R Thalamus \\
\hline & & -8 & -12 & 14 & $1 \mathrm{E}-02$ & $2 \mathrm{E}-02$ & 2.1 & L Thalamus \\
\hline
\end{tabular}


Table 2 continued

\begin{tabular}{|c|c|c|c|c|c|c|c|c|}
\hline \multirow[t]{2}{*}{ Cluster number } & \multirow[t]{2}{*}{ Volume $\left(\mathrm{mm}^{3}\right)$} & \multicolumn{3}{|c|}{ MNI coordinates } & \multirow[t]{2}{*}{ ALE } & \multirow[t]{2}{*}{$P$} & \multirow[t]{2}{*}{$z$} & \multirow[t]{2}{*}{ Label (Side region BA) } \\
\hline & & $x$ & $y$ & $z$ & & & & \\
\hline \multirow[t]{20}{*}{3} & \multirow[t]{20}{*}{17064} & -44 & -10 & -6 & $2 \mathrm{E}-02$ & $5 E-05$ & 3.9 & L Insula BA13 \\
\hline & & -42 & -16 & 0 & $2 \mathrm{E}-02$ & $2 \mathrm{E}-04$ & 3.5 & L Insula BA13 \\
\hline & & -36 & 22 & -2 & $1 \mathrm{E}-02$ & $2 \mathrm{E}-03$ & 2.9 & L Insula BA13 \\
\hline & & -40 & 12 & -2 & $1 E-02$ & $2 \mathrm{E}-03$ & 2.8 & L Insula BA13 \\
\hline & & -40 & -18 & 14 & $1 \mathrm{E}-02$ & $3 E-03$ & 2.8 & L Insula BA13 \\
\hline & & -48 & 2 & -8 & $1 \mathrm{E}-02$ & $4 \mathrm{E}-03$ & 2.7 & L Superior temporal gyrus BA22 \\
\hline & & -22 & 14 & -14 & $1 \mathrm{E}-02$ & $6 \mathrm{E}-03$ & 2.5 & L Lentiform nucleus \\
\hline & & -24 & 16 & -8 & $1 \mathrm{E}-02$ & $7 \mathrm{E}-03$ & 2.5 & L Lentiform nucleus \\
\hline & & -38 & 10 & -10 & $1 \mathrm{E}-02$ & $7 \mathrm{E}-03$ & 2.5 & L Insula BA13 \\
\hline & & -34 & -8 & -26 & $1 \mathrm{E}-02$ & $7 E-03$ & 2.5 & L Parahippocampal gyrus \\
\hline & & -46 & -2 & 2 & $1 \mathrm{E}-02$ & $9 \mathrm{E}-03$ & 2.4 & L Insula BA13 \\
\hline & & -42 & 12 & 6 & $1 \mathrm{E}-02$ & $9 \mathrm{E}-03$ & 2.4 & L Insula BA13 \\
\hline & & -54 & 18 & 2 & $1 \mathrm{E}-02$ & $1 \mathrm{E}-02$ & 2.3 & L Precentral gyrus BA44 \\
\hline & & -48 & 16 & -2 & $1 \mathrm{E}-02$ & $1 E-02$ & 2.3 & L Insula BA13 \\
\hline & & -64 & -4 & -16 & $1 \mathrm{E}-02$ & $1 E-02$ & 2.3 & L Middle temporal gyrus BA21 \\
\hline & & -58 & -4 & -16 & $1 \mathrm{E}-02$ & $1 \mathrm{E}-02$ & 2.3 & L Middle temporal gyrus BA21 \\
\hline & & -30 & 18 & -18 & $1 \mathrm{E}-02$ & $1 E-02$ & 2.3 & L Inferior frontal gyrus BA47 \\
\hline & & -60 & 2 & -4 & $1 \mathrm{E}-02$ & $1 \mathrm{E}-02$ & 2.2 & L Superior temporal gyrus \\
\hline & & -38 & -28 & 10 & $1 \mathrm{E}-02$ & $1 \mathrm{E}-02$ & 2.2 & L Transverse temporal gyrus BA41 \\
\hline & & -32 & 16 & -26 & $1 \mathrm{E}-02$ & $2 \mathrm{E}-02$ & 2.2 & L Inferior frontal gyrus BA47 \\
\hline \multicolumn{9}{|c|}{ c. GM: Higher volume with use (HC<SU) } \\
\hline \multirow[t]{6}{*}{1} & \multirow[t]{6}{*}{22,440} & -26 & -4 & 2 & $3 \mathrm{E}-02$ & $1 E-13$ & 7.3 & L Putamen \\
\hline & & -14 & 4 & -8 & $1 \mathrm{E}-02$ & $5 \mathrm{E}-06$ & 4.4 & L Lateral globus pallidus \\
\hline & & -28 & 8 & -8 & $1 \mathrm{E}-02$ & $4 \mathrm{E}-05$ & 4.0 & L Putamen \\
\hline & & -30 & 14 & 2 & $1 \mathrm{E}-02$ & $2 \mathrm{E}-04$ & 3.6 & L Claustrum \\
\hline & & -24 & -12 & -8 & $1 \mathrm{E}-02$ & $2 \mathrm{E}-04$ & 3.6 & L Lateral globus pallidus \\
\hline & & -16 & 20 & -16 & $9 \mathrm{E}-03$ & $5 \mathrm{E}-04$ & 3.3 & L Subcallosal Gyrus BA47 \\
\hline \multicolumn{9}{|l|}{ d. WM: All foci } \\
\hline \multirow[t]{9}{*}{1} & \multirow[t]{9}{*}{37,328} & 6 & -26 & -2 & $2 \mathrm{E}-02$ & $5 \mathrm{E}-07$ & 4.9 & R Anterior thalamic radiation \\
\hline & & 8 & -32 & 10 & $1 \mathrm{E}-02$ & $5 \mathrm{E}-05$ & 3.9 & R Corpus callosum \\
\hline & & -6 & -38 & -14 & $1 \mathrm{E}-02$ & $2 \mathrm{E}-04$ & 3.6 & L Corticospinal tract \\
\hline & & -12 & -28 & 8 & $1 \mathrm{E}-02$ & $2 \mathrm{E}-04$ & 3.5 & L Fornix \\
\hline & & -4 & -26 & -2 & $1 \mathrm{E}-02$ & $2 \mathrm{E}-04$ & 3.5 & L Anterior thalamic radiation \\
\hline & & -4 & 2 & 24 & $1 \mathrm{E}-02$ & $2 \mathrm{E}-04$ & 3.5 & L Corpus callosum \\
\hline & & 2 & 10 & 22 & $1 E-02$ & $2 \mathrm{E}-04$ & 3.5 & R Corpus callosum \\
\hline & & -2 & -30 & 12 & $1 \mathrm{E}-02$ & $2 \mathrm{E}-04$ & 3.5 & L Corpus callosum \\
\hline & & 6 & -22 & 12 & $1 E-02$ & $3 \mathrm{E}-04$ & 3.5 & R Fornix \\
\hline
\end{tabular}


Table 2 continued

\begin{tabular}{|c|c|c|c|c|c|c|c|c|}
\hline \multirow[t]{2}{*}{ Cluster number } & \multirow[t]{2}{*}{ Volume $\left(\mathrm{mm}^{3}\right)$} & \multicolumn{3}{|c|}{ MNI coordinates } & \multirow[t]{2}{*}{ ALE } & \multirow[t]{2}{*}{$P$} & \multirow[t]{2}{*}{$z$} & \multirow[t]{2}{*}{ Label (Side region BA) } \\
\hline & & $x$ & $y$ & $z$ & & & & \\
\hline & & 32 & -32 & 0 & $1 E-02$ & $3 E-04$ & 3.4 & R Optic radiation \\
\hline & & -16 & -42 & 2 & $1 E-02$ & $3 E-04$ & 3.4 & L Cingulum \\
\hline & & -4 & -14 & 18 & $1 \mathrm{E}-02$ & $3 E-04$ & 3.4 & L Fornix \\
\hline & & -8 & -28 & -30 & $1 E-02$ & $4 \mathrm{E}-04$ & 3.4 & $\mathrm{~L}$ Corticospinal tract \\
\hline & & 18 & -34 & 4 & $9 \mathrm{E}-03$ & $5 \mathrm{E}-04$ & 3.3 & R Fornix \\
\hline & & -16 & -28 & -18 & $9 \mathrm{E}-03$ & $5 \mathrm{E}-04$ & 3.3 & L Cingulum \\
\hline & & -30 & -14 & -8 & $9 \mathrm{E}-03$ & $9 \mathrm{E}-04$ & 3.1 & L Optic radiation \\
\hline & & -32 & -10 & -14 & $9 \mathrm{E}-03$ & $1 \mathrm{E}-03$ & 3.1 & L Optic radiation \\
\hline & & -22 & -24 & -8 & $8 \mathrm{E}-03$ & $1 \mathrm{E}-03$ & 3.0 & L Optic radiation \\
\hline \multicolumn{9}{|c|}{ e. WM: Lower volume with use $(\mathrm{SU}<\mathrm{HC})$} \\
\hline \multirow[t]{15}{*}{1} & 33,624 & 6 & -26 & -2 & $2 \mathrm{E}-02$ & $4 \mathrm{E}-07$ & 4.9 & $\mathrm{R}$ Anterior thalamic radiation \\
\hline & & 8 & -32 & 10 & $1 \mathrm{E}-02$ & $5 \mathrm{E}-05$ & 3.9 & R Corpus callosum \\
\hline & & -6 & -38 & -14 & $1 E-02$ & $1 E-04$ & 3.6 & $\mathrm{~L}$ Corticospinal tract \\
\hline & & -12 & -28 & 8 & $1 E-02$ & $2 \mathrm{E}-04$ & 3.6 & L Fornix \\
\hline & & -4 & -26 & -2 & $1 E-02$ & $2 \mathrm{E}-04$ & 3.5 & L Anterior thalamic radiation \\
\hline & & -4 & 2 & 24 & $1 E-02$ & $2 \mathrm{E}-04$ & 3.5 & L Corpus callosum \\
\hline & & 2 & 10 & 22 & $1 \mathrm{E}-02$ & $2 \mathrm{E}-04$ & 3.5 & R Corpus callosum \\
\hline & & -2 & -30 & 12 & $1 \mathrm{E}-02$ & $2 \mathrm{E}-04$ & 3.5 & L Corpus callosum \\
\hline & & 6 & -22 & 12 & $1 E-02$ & $2 \mathrm{E}-04$ & 3.5 & R Fornix \\
\hline & & 32 & -32 & 0 & $1 E-02$ & $3 E-04$ & 3.4 & $\mathrm{R}$ Optic radiation \\
\hline & & -16 & -42 & 2 & $1 \mathrm{E}-02$ & $3 \mathrm{E}-04$ & 3.4 & L Cingulum \\
\hline & & -4 & -14 & 18 & $1 \mathrm{E}-02$ & $3 E-04$ & 3.4 & L Fornix \\
\hline & & -8 & -28 & -30 & $1 E-02$ & $4 \mathrm{E}-04$ & 3.4 & L Corticospinal tract \\
\hline & & 18 & -34 & 4 & $9 \mathrm{E}-03$ & $4 \mathrm{E}-04$ & 3.3 & R Fornix \\
\hline & & -14 & -30 & -18 & $9 \mathrm{E}-03$ & $5 \mathrm{E}-04$ & 3.3 & L Cingulum \\
\hline \multicolumn{9}{|c|}{ f. WM: Higher volume with use $(H C<S U)$} \\
\hline 1 & 27,736 & 14 & -14 & -16 & $2 \mathrm{E}-04$ & $1 \mathrm{E}-02$ & 2.3 & R Corticospinal tract \\
\hline 2 & 14,712 & -46 & -6 & -30 & $8 \mathrm{E}-03$ & $2 \mathrm{E}-05$ & 4.2 & L Superior longitudinal fasciculus \\
\hline 3 & 14,712 & -22 & -24 & -8 & $8 \mathrm{E}-03$ & $2 \mathrm{E}-05$ & 4.2 & L Optic radiation \\
\hline
\end{tabular}

GM gray matter, WM white matter, SU substance user, $H C$ healthy control, $A L E$ anatomic likelihood estimation, $P p$-value, $Z$ peak $Z$-value, $R$ right, $L$ left.

\section{Subgroup analysis by type of substance}

In the second subgroup meta-analysis, we reported ALE maps of substance users (SU) against healthy controls (HC) by type of substance. Given that we only included one publication on ketamine, this substance was not included in the subgroup analysis. We found significant ALE maps showing lower GM volume in all substances, and higher GM volume only in tobacco, cannabis, and polysubstance. Also, we found lower WM volume in alcohol, tobacco and cocaine, and found no higher WM volume in any substance (Fig. 2 and Supplementary Table 5).

We conducted contrast analyses between the ALE maps of each subgroup, to determine similarity (conjunction) and/or difference (subtraction) of affected brain regions between the types of substance (Fig. 3 and Supplementary Table 6). Alcohol, overall, differed with most of the other substances including tobacco, cocaine, cannabis, and opioids. Conversely, cannabis shared affected areas with tobacco, opioids, stimulants, and 


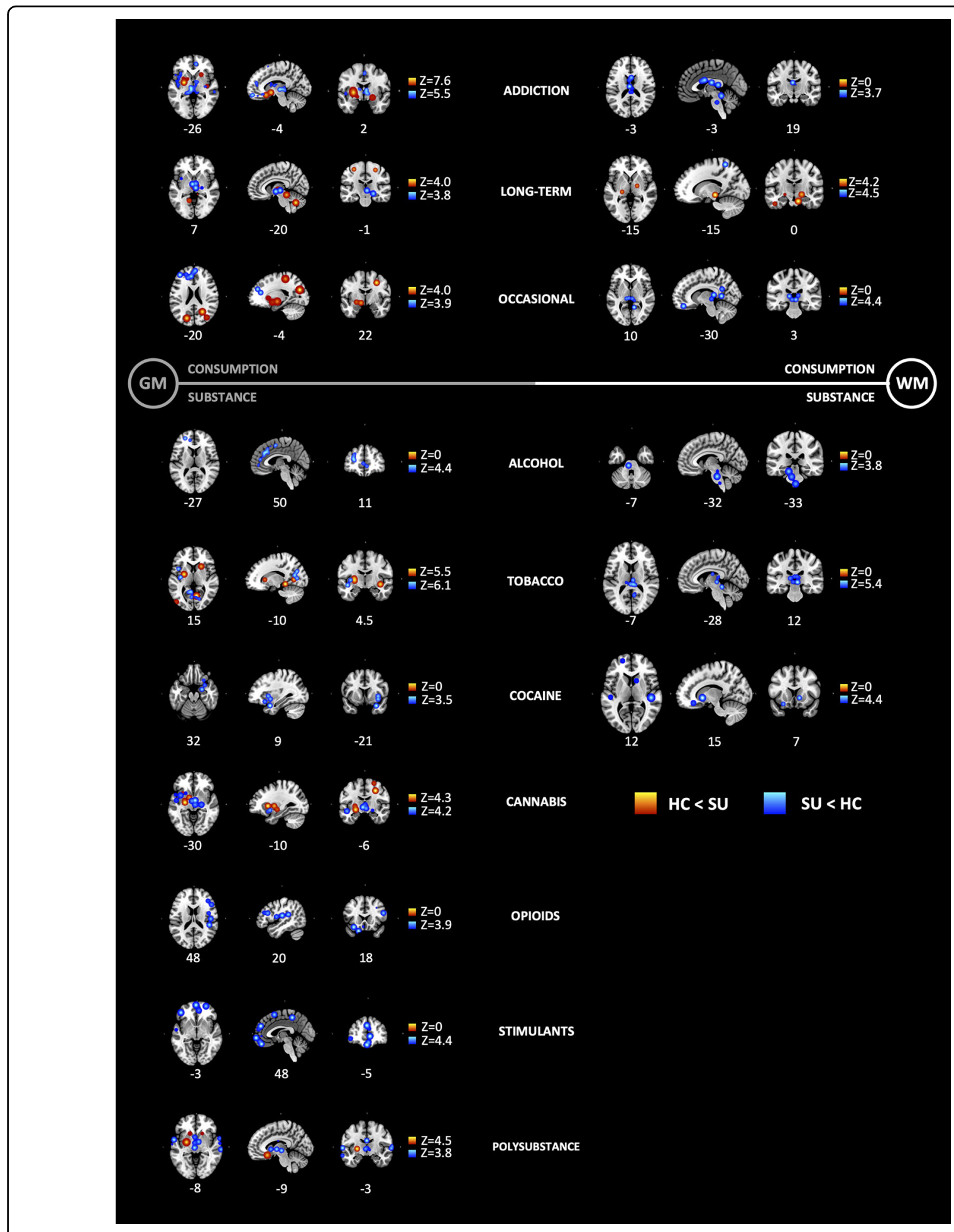

Fig. 2 Subgroup anatomic likelihood estimation meta-analytic results for studies comparing brain morphological changes between SU and $\mathrm{HC}$, at cluster level inference $\boldsymbol{p}<\mathbf{0 . 0 5}$ (FWE). Subgroup analyses included consumption severity (top) and type of substance (bottom). HC $<$ $\mathrm{SU}=$ higher volume with use; $\mathrm{SU}<\mathrm{HC}=$ lower volume with use. Significant ALE maps showing lower GM and WM volumes across all types of consumption; higher GM volumes were also shown across all types of consumption; higher WM only in long-term use; lower GM volume in all substances, and higher GM volume only in tobacco, cannabis and polysubstance; lower WM volume in alcohol, tobacco and cocaine, and found no higher WM volume in any substance. Consumption: addiction $(k=49)$ vs. long-term use $(k=5)$ vs. occasional use $(k=6)$. Substance: alcohol $(k=14)$ vs. tobacco $(k=13)$ vs. cannabis $(k=7)$ vs. cocaine $(k=7)$ vs. stimulants $(k=3)$ vs. opioids $(k=8)$ vs. ketamine $(k=1)$, and papers that pooled together substances which we termed polysubstance $(k=7)$. $Z$, peak $Z$-value. 


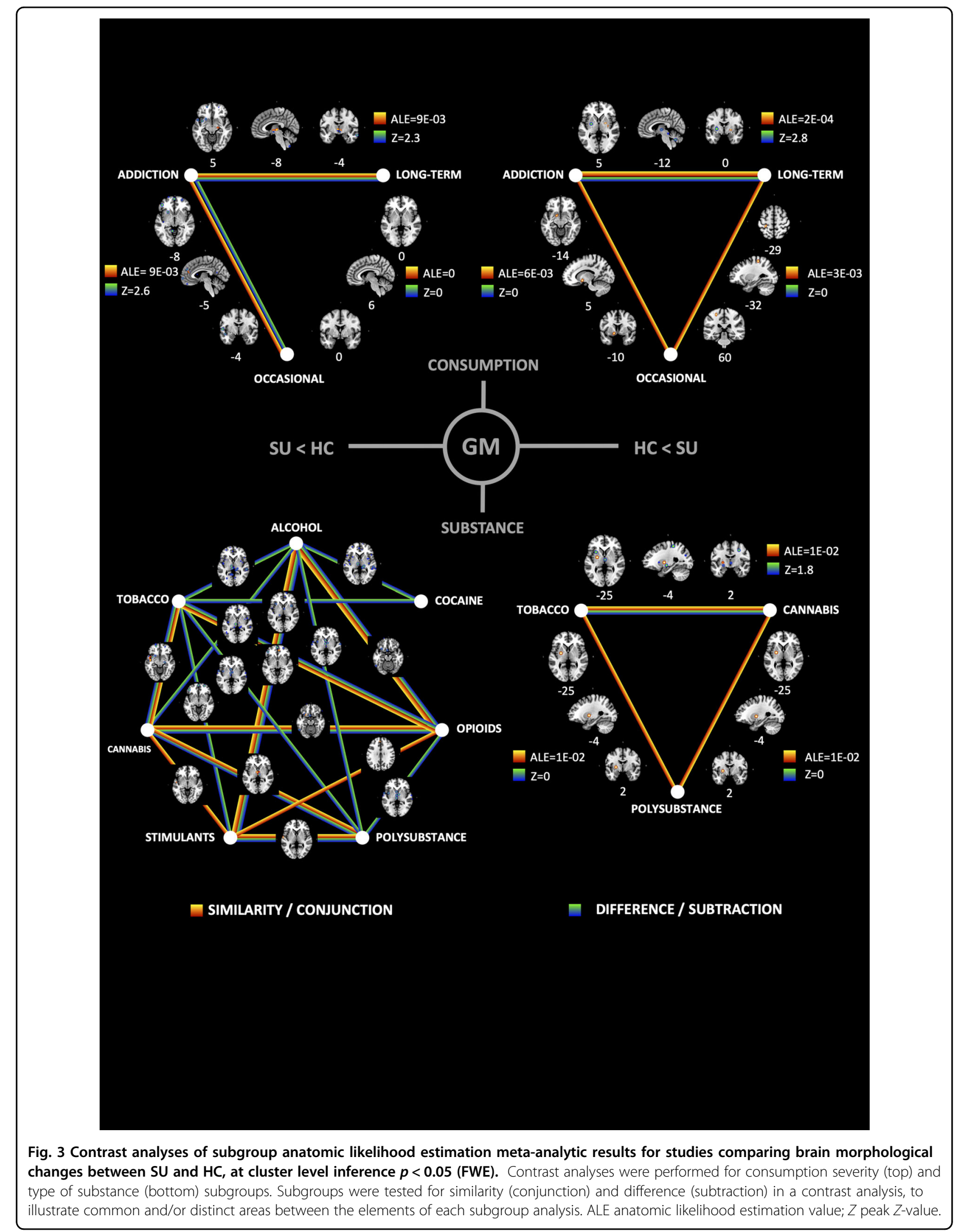


polysubstance. Consistent affected shared areas included thalamus, insula, inferior frontal gyrus, and superior temporal gyrus in GM; and anterior thalamic radiation in WM. Although most addictive substances share a common neurobiological process in the reward circuitry, it is evident that neuroadaptations in SUD depend on the type of substance used. Results of this subgroup analysis by substance is valuable for future research into the best approach for therapeutics (pharmacological and behavioral), as treatment effects can be correlated with brain morphometry.

\section{Discussion}

In this systematic review and meta-analysis, we used coordinate-based anatomic likelihood estimation (ALE) to pool the effects of substance use disorders (SUDs) on brain regional volume. We found that the most converging regions with volume pathology in SUDs were putamen, thalamus, insula and anterior cingulate cortex in gray matter (GM), and the thalamic radiation, corticospinal tract, and corpus callosum in white matter (WM). We found that consumption severity and type of substance subgroups resulted in significant ALE maps with both shared and distinctive regions involved, supporting converging and divergent effects depending on severity and type of substance use.

\section{Characteristics of the included studies}

Overall, the included publications clearly stated their research question, population, inclusion and exclusion criteria, measurements, and outcomes. We found that most of the publications failed to report the type of evaluator (e.g., psychiatrist), and some did not mention if the DSM or other diagnostic criteria were used to diagnose SUD. In terms of MRI characteristics and quality of the studies, we found that all included studies used state-ofthe-art techniques and statistical tools, and therefore support the standardization of neuroimaging studies as a key element in future research and reproducibility efforts $^{31-33}$. However, a larger effort is needed to provide diagnosis criteria, which would result in improved classifications for future reviews and meta-analyses.

\section{Primary outcome: altered brain morphometry in SUDs}

SUDs seems to disrupt the normal function of the limbic loop of the basal ganglia ${ }^{3}$. Neuroplastic adaptations in cortical and subcortical regions seem to progress with the severity of the SUD ${ }^{46}$. However, the relation between repeated dopaminergic signaling in the basal ganglia and volumetric alterations is still unclear, thus, causality should be interpreted with caution. Indeed, we report consistent volumetric alterations in putamen, thalamus, insula, and anterior cingulate cortex in GM, and internal capsule and thalamic radiations in WM, supporting the idea that the entire limbic loop of the basal ganglia shows neuroadaptations associated to SUDs.

Higher putamen volume may be explained by the repeated glutamatergic spikes onto dopamine neurons (VTA/SNc) and into MSN in dorsal and ventral striatum, supported by behavioral changes in reward responsivity and habituation that characterize SUDs. Notably, almost all regions of the neocortex project direct input to the striatum. Most of these projections come from association areas in frontal and parietal lobes, with contributions from temporal, insular, and cingulate cortices. These projections (corticostriatal pathway) travel via the internal capsule to reach the caudate and putamen $^{47}$. We also found higher WM volume of the internal capsule in SUDs, suggesting neuroadaptive processes in this pathway as well. It has been suggested that SUDs or addiction are a disease of self-control ${ }^{48}$. Although the study of SUDs has been focused mainly on the role of dopamine and the reward system, new findings of clinical studies have revealed neuroplastic mechanisms in frontocortical regions that may underlie reward-seeking behavior ${ }^{13}$. In susceptible individuals, certain stimuli may activate strong urges that are not congruent with a given context. The lack of a proper inhibitory control may keep these urges in control up to a point, when stronger impulses and deficient inhibition result in impulsive or compulsive behavior ${ }^{49}$. Current models of SUDs suggest that impulsivity and compulsivity characterize the pathological behavior and help explain our structural results ${ }^{3}$.

It has been proposed that the insula and the anterior cingulate cortex form the salience network (SN), that coordinates between the default mode network (DMN) and the central executive network $(\mathrm{CEN})^{50}$. In our study we found lower volume of the insula, a region whose morphology has been associated with substance use compulsion and severity ${ }^{51}$. The insula plays a major role in interoception by integrating information from the internal physiological state, and projecting information to the ACC, ventral striatum and prefrontal cortex to initiate adaptive responses ${ }^{52}$. In SUDs, the insula's ability to switch between networks seems to be affected, as well as its functional connectivity with the ACC, amygdala and putamen $^{53-55}$. Similarly, SUD neuroimaging studies have shown disrupted activity of the $\mathrm{ACC}^{3}$, involved in inhibitory control $^{56}$, and altered connectivity with the insula $^{53}$. The rostral part of the ACC is implicated in error-related responses, including affective processing, and the caudal part of the ACC is associated with detection of conflict to recruit cognitive control ${ }^{57}$. Thus, reduction in inputs from prefrontal and cingulate cortices into striatum may disrupt the control over action selec$\operatorname{tion}^{58}$ (see Supplementary Table 7 for MACM, and Supplementary Table 8 for functional characterization). 
Finally, we found that SUDs were associated with lower thalamic GM/WM across several substances including alcohol, cocaine, nicotine, methamphetamine, opioids, and cannabis ${ }^{59,60}$. Reduced structural and functional integrity of the thalamus and its connectivity appear to be associated with the severity of SUD ${ }^{61}$. Overall, there are brain regions consistently affected in all SUDs, with diverging MRI manifestations (higher vs. lower volume) suggesting different underlying structural pathology between brain regions.

\section{Common and distinct patterns of brain volume alterations across consumption severity}

The effect of substance use in the brain seems to vary across the severity of consumption. Cortical structures seem affected in occasional use, while established addictive consumption (addiction) seems to also affect subcortical regions of the brain such as thalamus and basal ganglia. Such disrupted GM areas may presumably be coaffected with its respective WM thalamic radiation and corpus callosum connection, as seen in our results. Occasional use seems to affect WM tracts of the cingulum, connecting the limbic system with areas such as the cingulate gyrus, entorhinal cortex, and temporal lobe. Neuroimaging studies have found that disruption of the posterior cingulum is associated to cognitive impairment $^{62}$. The forceps minor connects the lateral and medial surfaces of the frontal lobes and crosses the midline via the genu of the corpus callosum ${ }^{47}$, and also showed structural alterations in occasional use. Along with the anterior thalamic radiation, the forceps minor connects ACC and striatum to the anterior frontal regions, modulating executive functions ${ }^{63}$.

Various physiological mechanisms such as oxidative stress, mitochondrial dysfunction, or neurotrophic factor dysfunction might account for the observed cortical GM volume reductions in occasional use ${ }^{64}$. Presumably, repeated dopaminergic stimulation from substance abuse produce neuroadaptations (e.g., dendritic morphology and ionotropic glutamate receptors), that result in longterm potentiation (LTP) and long-term depression $(\text { LTD })^{11}$ of the basal ganglia neurocircuitry ${ }^{3}$. These results suggest that cortical morphological pathology in SUDs appears before subcortical pathology or that subcortical pathology is only seen when addiction is established. This needs to be explored further with longitudinal studies.

\section{Common and distinct patterns of brain volume alterations across types of substances}

Reward processes are shared between substances, namely repeated stimulation into the VTA which releases dopamine into the ventral striatum ${ }^{3}$. However, the stimulation of the mesolimbic system depends on the different molecular targets for each kind of substance. For example, alcohol, unlike most other drugs, affects a wide range of targets and indirectly increases dopamine in the $\mathrm{NAc}^{65}$. Stimulants like amphetamine and cocaine block dopamine transporters, thus increasing dopamine in $\mathrm{NAc}^{66}$. Cannabis activates receptors that release neurotransmitters (GABA/ Glutamate), modulating the activity of the mesolimbic system. Opioids, agonists of mu opioid receptors (MOR) in VTA, increase striatal dopamine release ${ }^{67}$. Nicotine and its interactions with nicotinic acetylcholine receptors, increases neuronal activity in $\mathrm{VTA}^{68}$. In our results, most of the substances show a convergent effect and region, namely lower volume of the thalamus.

Divergently, alcohol seems to affect frontal areas including superior and medial frontal gyrus, as well as ACC. Tobacco use shows a myriad of alterations including lower volume in insula and posterior areas of the DMN, such as PCC and precuneus. Cocaine users show lower volume of the claustrum, a structure that connects prefrontal areas with the thalamus, and has close proximity to the insula and putamen ${ }^{69}$. Cannabis use reduces the volume of temporal areas and thalamus, and increases the volume of putamen, while opioid use affects cortical fronto-temporal areas. Stimulant use mainly reduces GM volume of the frontal lobe. Polysubstance studies, as expected, show a wide variety of affected areas including lower volume of the anterior cingulate gyrus, thalamus, and superior temporal gyrus, and show higher volume of the subcallosal gyrus. In terms of WM, the affected convergent regions were the corticospinal tract, anterior thalamic radiation, the corpus callosum, and the cingulum. Overall, different substances show convergent and divergent morphological pathology, suggesting different physiopathology and possibly therapeutic approaches in SUDs that need to be considered.

\section{Limitations and future perspectives}

A comprehensive review of the current kind is valuable in both synthesizing the effect of SUD on brain morphometry and highlighting issues in the field for future perspectives. For example, by setting a clear contrast based on MRI paradigms (e.g., SU < HC), we try to narrow the heterogeneity inherent to SUD as we relied on two assumptions: (1) contrasts we pool are based on best practices and (2) the Ginger ALE method. To conduct the ALE meta-analysis, we pooled peak coordinates derived from the included studies, rather the original raw structural MRI images. The accuracy of our findings relies on the result of a statistical estimation of coordinate-based anatomic foci (input), treated as spatial probability distributions centered at the given coordinates. Consequently, the individual profiles of substance users are not included in this review, and future syntheses might examine individual participant-level data, as these become increasingly available. For example, a number of studies may investigate a primary substance, but individuals 
within such studies may consume other substances as well, and are not listed as polysubstance users.

Notably, we included only VBM studies, and recognize that other methods measuring brain structure may provide more accurate morphometric results. However, a whole-brain approach is an important requisite in coordinate-based meta-analyses ${ }^{70}$, in which anatomic convergence across experiments is tested under the assumption that each voxel has a priori the same chance of being significant. Thus, inclusion of heterogeneous analyses such as region-of-interest (ROIs) or small volume corrected (SVC) analyses would violate such assumption and lead to overrepresentation of those regions. Likewise, VBM studies on WM are not as precise as, for example, diffusion-weighted imaging (DWI).

The heterogeneity among the methods used in the included studies, such as preprocessing software, smoothing, statistical thresholds, participants' characteristics, medication history and comorbidity, represent additional confounders. Meta-regression analysis is not compatible with GingerALE. We therefore did not perform regression-based assessments of factors that might be implicated in heterogeneity (e.g., age of participants, age of first use, and total years of SUD).

As traditional meta-analyses, coordinate-based metaanalyses such as ALE can be subject to different forms of publication bias which may impact results and invalidate findings (e.g., the "file drawer problem"). We performed the Fail-Safe $\mathrm{N}$ analysis (FSN) ${ }^{43}$ as a measure of robustness against potential publication bias. It is estimated for normal human brain mapping that a 95\% confidence interval for the number of studies that report no local maxima varies from 5 to 30 per 100 published studies. In our study, we tested 11 clusters resulting from our primary outcomes. We found that all clusters showed an FNR greater or equal than the minimum imposed of 18 . FNR was $>350$ for clusters resulting from all GM foci analysis, and $>300$ for clusters resulting from all WM foci analysis. Thus, indicating a robust convergence of foci in these regions but also indicating that proportionally fewer studies are needed to obtain this effect. Two clusters from the comparison GM SU $<\mathrm{HC}$ showed an FSN between the lower and upper boundary (Supplementary Table 9).

In our review, the included studies did not acquire the long-term measurements necessary to show that SUD is temporally linked to a decrease or increase of brain tissue, as a longitudinal design might provide; but they rather examine brain morphometry in established substance users compared to non-users. Socio-economic and educational background data on participants are lacking in most of the studies, limiting the potential for statistical correction using naturalistic environmental confounders.

We conducted a sensitivity analysis on primary outcomes, excluding $1.5 \mathrm{~T}$ studies (Supplementary Table 10).
Potentially, studies using different field strength scanners may result in varying levels of signal-to-noise and contrast-to-noise ratios. As the ALE method relies only on coordinates reported on the included studies, the fields strength is not considered and may affect the results. No differences with the main results were identified.

In the consumption and substance subgroup analyses, the number of experiments for each category of the subgroup analyses was unmatched (e.g., addiction 64\%, occasional use $29 \%$, and long-term use $7 \%$ ). Although the ALE method weights the result on the number of participants per experiment, the resulting ALE maps of subgroup and contrast analyses should be interpreted with caution.

The progression from initial drug use to established SUD may depend on age and developmental stage ${ }^{71}$. Critical periods of development are characterized by functional neuroplastic mechanisms that may be easily altered by pathological neuroadaptations due to $\mathrm{SUD}^{5}$. For example, delays in maturation associated with drug exposure, genetics, or social environment, may increase risky behaviors in adolescents ${ }^{72}$. Brain imaging studies have found altered structure of prefrontal cortices associated with higher risk for SUD in adolescents ${ }^{73}$, suggesting that control executive functions such as decision making and impulse control (inhibition) are immature ${ }^{74}$. Unfortunately, the neurobiological underpinnings of neuroadaptations for both functional development and SUD, are not fully understood, in part, by a high variability in VBM results ${ }^{75}$. In this review, the included studies failed to report enough experiments (foci $<15$ ), to conduct an age subgroup analysis (e.g., adolescents vs. adults).

SUDs are frequently co-diagnosed with psychiatric and neurological disorders (Common comorbidities with substance use disorders). Research suggests that adolescents with SUD have high rates of co-occurring mental illness, up to $60 \%{ }^{76}$. The most common psychiatric comorbidities with SUD include anxiety disorders, posttraumatic stress disorder, depression, bipolar disorder, attention-deficit hyperactivity disorder, psychosis, borderline disorder, and schizophrenia. Notably, establishing causality or directionality between mental illness and SUD is difficult, however, common risk factors are shared ${ }^{77}$. Additionally, recent research has focused on the neurological effects of SUD, rather than as comorbid, cooccurring alterations ${ }^{78}$ (e.g., SUD and Parkinson's disease). In this review, the included studies failed to report enough experiments (foci $<15$ ), to conduct a comorbidity subgroup analysis (e.g., pure addiction vs. comorbid addiction). Nevertheless, it is important to recognize that mental illness and SUD share alterations in the same neurotransmitter systems (e.g., dopaminergic ${ }^{4}$ ) and in brain areas involved in reward, decision making, impulse control, and emotion ${ }^{79}$. 


\section{Conclusions}

In conclusion, the present systematic review and metaanalysis of voxel-based morphometry neuroimaging studies provides evidence of common and distinct morphological gray matter and white matter pathology in substance use disorders. We found consistent morphometric alterations in regions of the insula, anterior cingulate cortex, basal ganglia (putamen), and thalamus, with their respective white matter thalamic radiation and internal capsule bundle. Our subgroup analysis showed distinct volume alterations depending on the type of consumption (occasional vs. long-term vs. addiction) and type of substance. This evidence may help future studies to better understand substance use disorders and possible new therapeutic approaches.

\section{Acknowledgements}

Scholarship for Sofia Fernandez-Lozano funded by CONACYT No. 476284. This study was supported by public fund CONACYT FOSISS No. 0260971.

\begin{abstract}
Author details
${ }^{1}$ Department of Clinical Medicine, Center for Music in the Brain, University of Aarhus, Aarhus, Denmark. ${ }^{2}$ Instituto de Neurobiología, Universidad Nacional Autónoma de México (UNAM) campus Juriquilla, Queretaro, Mexico. ${ }^{3}$ Instituto Nacional de Psiquiatría "Ramón de la Fuente Muñiz", Mexico City, Mexico. ${ }^{4}$ Facultad de Medicina, Universidad Nacional Autónoma de México (UNAM), Mexico City, Mexico. ${ }^{5}$ Facultad de Psicología, Universidad Nacional Autónoma de México (UNAM), Mexico City, Mexico. ${ }^{6}$ Department of Clinical Medicine, Interacting Minds Center, University of Aarhus, Aarhus, Denmark. ${ }^{7}$ Department of Clinical Medicine, Center of Functionally Integrative Neuroscience, University of Aarhus, Aarhus, Denmark
\end{abstract}

\section{Conflict of interest}

The authors declare that they have no conflict of interest.

\section{Publisher's note}

Springer Nature remains neutral with regard to jurisdictional claims in published maps and institutional affiliations.

Supplementary Information accompanies this paper at (https://doi.org/ 10.1038/s41398-020-01128-2).

Received: 4 June 2020 Revised: 10 November 2020 Accepted: 20 November 2020

Published online: 11 January 2021

\section{References}

1. Association, A. P. Diagnostic and Statistical Manual of Mental Disorders 5th edn (American Psychiatric Association, Washington, 2013).

2. United Nations Office on Drugs and Crime. World Drug Report 2019. (UN, 2019). https://doi.org/10.18356/a4dd519a-en.

3. Koob, G. F. \& Volkow, N. D. Neurocircuitry of addiction. Neuropsychopharmacology 35, 217-238 (2009).

4. Volkow, N. D., Fowler, J. S., Wang, G. J., Baler, R. \& Telang, F. Imaging dopamine's role in drug abuse and addiction. Neuropsychopharmacology https:// doi.org/10.1016/j.neuropharm.2008.05.022 (2009).

5. Volkow, N. D., Michaelides, M. \& Baler, R. The neuroscience of drug reward and addiction. Physiol. Rev. 99, 2115-2140 (2019).

6. Crow, T. J. Catecholamine-containing neurones and electrical self-stimulation: 2. A theoretical interpretation and some psychiatric implications. Psychol. Med. 3, 66-73 (1973)

7. Everitt, B. J. \& Robbins, T. W. Drug addiction: updating actions to habits to compulsions ten years on. Annu. Rev. Psychol. 67, 23-50 (2016).
8. Vink, J. M. Genetics of addiction: future focus on gene $\times$ environment interaction? J. Stud. Alcohol Drugs https://doi.org/10.1037/a0017376 (2016).

9. Mitchell, M. R., Berridge, K. C. \& Mahler, S. V. Endocannabinoid-enhanced "'Liking"' in nucleus accumbens shell hedonic hotspot requires endogenous opioid signals. Cannabis Cannabinoid Res. https://doi.org/10.1089/ can.2018.0021 (2018).

10. Grueter, B. A., Rothwell, P. E., Malenka, R. C., Sheng, M. \& Triller, A. Integrating synaptic plasticity and striatal circuit function in addiction: this review comes from a themed issue on Synaptic structure and function Edited. Curr. Opin. Neurobiol. 22, 545-551 (2012).

11. Kauer, J. A. \& Malenka, R. C. Synaptic plasticity and addiction. Nat. Rev. Neurosci. 8, 844-858 (2007)

12. Fowler, J. S., Volkow, N. D., Kassed, C. A. \& Chang, L. Imaging the addicted human brain. Science \& practice perspectives/a publication of the National Institute on Drug Abuse. Natl Inst. Health 3, 4-16 (2007).

13. Goldstein, R. Z. \& Volkow, N. D. Drug addiction and its underlying neurobiological basis: neuroimaging evidence for the involvement of the frontal cortex NIH public access. Am. J. Psychiatry 159, 1642-1652 (2002).

14. Demirakca, T. et al. Effects of alcoholism and continued abstinence on brain volumes in both genders. Alcoholism 35, 1678-1685 (2011).

15. Kühn, S. et al. Brain grey matter deficits in smokers: focus on the cerebellum. Brain Struct. Funct. 217, 517-522 (2012)

16. Almeida, O. P. et al. Smoking is associated with reduced cortical regional gray matter density in brain regions associated with incipient Alzheimer disease. Am. J. Geriatr. Psychiatry 16, 92-98 (2008).

17. Battistella, G. et al. Long-term effects of Cannabis on brain structure giovanni. Neuropsychopharmacology 39, 2041-2048 (2014).

18. Matochik, J. A., Eldreth, D. A., Cadet, J.-L. \& Bolla, K. I. Altered brain tissue composition in heavy marijuana users. Drug Alcohol Depend. 77, 23-30 (2005).

19. Matochik, J. A., London, E. D., Eldreth, D. A., Cadet, J.-L. \& Bolla, K. I. Frontal cortical tissue composition in abstinent cocaine abusers: a magnetic resonance imaging study. Neuroimage 19, 1095-1102 (2003).

20. Gallinat, J. et al. Smoking and structural brain deficits: a volumetric MR investigation. Eur. J. Neurosci. 24, 1744-1750 (2006).

21. Sim, M. E. et al. Cerebellar gray matter volume correlates with duration of cocaine use in cocaine-dependent subjects. Neuropsychopharmacology 32, 2229-2237 (2007)

22. $\mathrm{Bu}$, L. et al. Functional connectivity abnormalities of brain regions with structural deficits in young adult male smokers. Front. Hum. Neurosci. https:// doi.org/10.3389/fnhum.2016.00494 (2016)

23. Wetherill, R. R. et al. Cannabis, cigarettes, and their co-occurring use: disentangling differences in gray matter volume. Int. J. Neuropsychopharmacol. https://doi.org/10.1093/ijnp/pyv061 (2015).

24. Aoki, Y. et al. Volume reductions in frontopolar and left perisylvian cortices in methamphetamine induced psychosis. Schizophr. Res. 147, 355-361 (2013).

25. Chanraud, S. et al. Brain morphometry and cognitive performance in detoxified alcohol-dependents with preserved psychosocial functioning. Neuropsychopharmacology https://doi.org/10.1038/sj.npp.1301219 (2007).

26. Jang, D.P. et al. The relationship between brain morphometry and neuropsychological performance in alcohol dependence. Neurosci. Lett. 428, 21-26 (2007).

27. Gipson, C. D., Kupchik, Y. M. \& Kalivas, P. W. Rapid, transient synaptic plasticity in addiction. Neuropharmacology 76, 276-286 (2014).

28. Higgins, J. P. \& Green, S. Cochrane Handbook for Systematic Reviews of Interventions. (Wiley, Hoboken, 2011).

29. Moher, D., Liberati, A., Tetzlaff, J., Altman, D. G. \& Group, T. P. Preferred reporting items for systematic reviews and meta-analyses: the PRISMA statement. PLoS Med. 6, 1-5 (2009).

30. Covidence Systematic Review Software (Veritas Health Innovation, Melbourne). https://support.covidence.org/help/how-can-i-cite-covidence.

31. Poldrack, R. A. et al. Guidelines for reporting an fMRI study. Neuroimage 40, 409-414 (2008)

32. Tahmasian, M. et al. Practical recommendations to conduct a neuroimaging meta-analysis for neuropsychiatric disorders. Hum. Brain Mapp. 40, 5142-5154 (2019).

33. Nichols, T. E. et al. Best practices in data analysis and sharing in neuroimaging using MRI. Nat. Neurosci. 20, 299-303 (2017).

34. Eickhoff, S. B., Bzdok, D., Laird, A. R., Kurth, F. \& Fox, P. T. Activation likelihood estimation meta-analysis revisited. Neuroimage 59, 2349-2361 (2012).

35. Laird, A. R. et al. Comparison of the disparity between Talairach and MNI coordinates in functional neuroimaging data: validation of the Lancaster 
transform. Neuroimage https://doi.org/10.1016/j.neuroimage.2010.02.048 (2010).

36. Eickhoff, S. B. et al. Coordinate-based activation likelihood estimation metaanalysis of neuroimaging data: a random-effects approach based on empirical estimates of spatial uncertainty. Hum. Brain Mapp. https://doi.org/10.1002/ hbm.20718 (2009)

37. Fox, P. T. et al. User Manual for GingerALE 2.3 (Research Imaging Institute, UT Health Science Center, San Antonio, 2013).

38. Robinson, J. L., Laird, A. R., Glahn, D. C., Lovallo, W. R. \& Fox, P. T. Metaanalytic connectivity modeling: delineating the functional connectivity of the human amygdala. Hum. Brain Mapp. 31, 173-184 (2010).

39. Laird, A. R. et al. Networks of task co-activations. Neuroimage 80, 505-514 (2013).

40. Laird, A. R. et al. Investigating the Functional Heterogeneity of the Default Mode Network Using Coordinate-Based Meta-Analytic Modeling. https:/doi. org/10.1523/JNEUROSCl.4004-09.2009 (2019).

41. Gorgolewski, K. J. et al. NeuroVault.org: a web-based repository for collecting and sharing unthresholded statistical maps of the human brain. Front. Neuroinform. 9, 1-9 (2015).

42. Jenkinson, M., Beckmann, C. F., Behrens, T. E. J., Woolrich, M. W. \& Smith, S. M. FSL. Neuroimage 62, 782-790 (2012).

43. Acar, F., Seurinck, R., Eickhoff, S. B. \& Moerkerke, B. Assessing robustness against potential publication bias in activation likelihood estimation (ALE) metaanalyses for fMRI. PLOS ONE 13, e0208177 (2018).

44. Friston, K. et al. Statistical parametric maps in functional imaging: a general linear approach. Hum. Brain Mapp. 2, 189-210 (1995).

45. Cox, R. W. AFNl: software for analysis and visualization of functional magnetic resonance neuroimages. Comput. Biomed. Res. 29, 162-173 (1996).

46. Morales, M. \& Pickel, V. M. Insights to drug addiction derived from ultrastructural views of the mesocorticolimbic system. Ann. N. Y. Acad. Sci. 1248 71-88 (2012)

47. Haber, Suzanne N. "Corticostriatal Circuitry." Dialogues Clin. Neurosci. 18, 7-21 (2016).

48. Volkow, N. D. \& Baler, R. Addiction: a disease of self-control. Neurosci. Hum. Person 1-6 (2013).

49. Dalley, J. W., Everitt, B. J. \& Robbins, T. W. Impulsivity, compulsivity, and topdown cognitive control. Neuron 69, 680-694 (2011).

50. Menon, V. \& Uddin, L. Q. Saliency, switching, attention and control: a network model of insula function. Brain Struct. Funct. 214, 655-667 (2010).

51. Betka, S. et al. Signatures of alcohol use in the structure and neurochemistry of insular cortex: a correlational study. Psychopharmacology 236, 2579-2591 (2019).

52. Paulus, M. P., Tapert, S. F. \& Schulteis, G. The role of interoception and alliesthesia in addiction. Pharmacol. Biochem. Behav. 94, 1-7 (2009).

53. Droutman, V., Read, S. J. \& Bechara, A. Revisiting the role of the insula in addiction. Trends Cogn. Sci. 19, 414-420 (2015).

54. Naqvi, N. H. \& Bechara, A. The hidden island of addiction: the insula. Trends Neurosci. https://doi.org/10.1016/j.tins.2008.09.009 (2009).

55. Liang, X. et al. Interactions between the salience and default-mode networks are disrupted in cocaine addiction. J. Neurosci. 35, 8081-8090 (2015).

56. Volkow, N. D., Fowler, J. S. \& Wang, G.J. The addicted human brain viewed in the light of imaging studies: brain circuits and treatment strategies. Neuropharmacology 47, 3-13 (2004)

57. Tang, Y. Y., Posner, M. I., Rothbart, M. K. \& Volkow, N. D. Circuitry of self-control and its role in reducing addiction. Trends Cogn. Sci. 19, 439-444 (2015).

58. Renteria, R., Baltz, E. T. \& Gremel, C. M. Chronic alcohol exposure disrupts topdown control over basal ganglia action selection to produce habits. Nat. Commun. 9, 1-11 (2018)
59. Nurmedov, S. et al. Thalamic and cerebellar gray matter volume reduction in synthetic cannabinoids users. Eur. Addict. Res. 21, 315-320 (2015).

60. Wilson, B. A., Winegardner, J., Heugten, C. M. V. \& Ownsworth, T. (Eds.). Neuropsychological Rehabilitation: The International Handbook, 1st edn. (Routledge 2017) https://doi.org/10.4324/9781315629537.

61. Garza-Villarreal, E. A. et al. The effect of crack cocaine addiction and age on the microstructure and morphology of the human striatum and thalamus using shape analysis and fast diffusion kurtosis imaging. Transl. Psychiatry 7, e1122 (2017).

62. Bubb, E. J., Metzler-Baddeley, C. \& Aggleton, J. P. The cingulum bundle: anatomy, function, and dysfunction. Neurosci. Biobehav. Rev. 92, 104-127 (2018).

63. Mamiya, P. C., Richards, T. L. \& Kuhl, P. K. Right forceps minor and anterior thalamic radiation predict executive function skills in young bilingual adults. Front. Psychol. 9, 118 (2018).

64. Yamamoto, B. K. Moszczynska, A. \& Gudelsky, G. A. Amphetamine toxicities. Ann. N. Y. Acad. Sci. 1187, 101-121 (2010).

65. Wiers, C. E., Cabrera, E., Skarda, E., Volkow, N. D. \& Wang, G. J. PET imaging for addiction medicine: from neural mechanisms to clinical considerations. Prog. Brain Res. 224, 175-201 (2016).

66. Kahlig, K. M. \& Galli, A. Regulation of dopamine transporter function and plasma membrane expression by dopamine, amphetamine, and cocaine. Eur. J. Pharmacol. 479, 153-158 (2003).

67. Macey, T. A., Lowe, J. D. \& Chavkin, C. Mu opioid receptor activation of ERK1/2 is GRK3 and arrestin dependent in striatal neurons. J. Biol. Chem. 281, 34515-34524 (2006)

68. Bertrand, D. \& Terry, A. V. The wonderland of neuronal nicotinic acetylcholine receptors. Biochem. Pharmacol. 151, 214-225 (2018).

69. Chau, A., Salazar, A. M., Krueger, F., Cristofori, I. \& Grafman, J. The effect of claustrum lesions on human consciousness and recovery of function. Conscious. Cogn. 36, 256-264 (2015).

70. Müller, V. I. et al. Ten simple rules for neuroimaging meta-analysis. Neurosci. Biobehav. Rev. 84, 151-161 (2018).

71. Glisky, E. L. Changes in cognitive function in human aging. Brain Aging https:// doi.org/10.1201/9781420005523-1 (2019).

72. Guerri, C. \& Pascual, M. Mechanisms involved in the neurotoxic, cognitive, and neurobehavioral effects of alcohol consumption during adolescence. https:// doi.org/10.1016/j.alcohol.2009.10.003 (2010).

73. Jones, S. A., Morales, A. M., Lavine, J. B. \& Nagel, B. J. Convergent neurobiological predictors of emergent psychopathology during adolescence. Birth Defects Res. 109, 1613-1622 (2017).

74. Winters, K. C., Tanner-Smith, E. E., Bresani, E. \& Meyers, K. Current advances in the treatment of adolescent drug use. Adolesc. Health. Med. Ther. https://doi. org/10.2147/AHMT.S48053 (2014).

75. Gogtay, N. \& Thompson, P. M. Mapping gray matter development: implications for typical development and vulnerability to psychopathology. Brain Cogn. 72, 6-15 (2010).

76. Hser, Y. I. et al. An evaluation of drug treatments for adolescents in 4 US cities. Arch. Gen. Psychiatry 58, 689-695 (2001).

77. Cerdá, M., Sagdeo, A., Johnson, J. \& Galea, S. Genetic and environmental influences on psychiatric comorbidity: a systematic review. J. Affect. Disord. 126, 14-38 (2010)

78. McHugo, G. J. et al. The prevalence of traumatic brain injury among people with co-occurring mental health and substance use disorders. J. Head. Trauma Rehabil. 32, E65-E74 (2017).

79. Wing, V. C., Wass, C. E., Soh, D. W. \& George, T. P. A review of neurobiological vulnerability factors and treatment implications for comorbid tobacco dependence in schizophrenia. Ann. N. Y. Acad. Sci. 1248, 89-106 (2012). 